\title{
3-Aminoiminoacrylate, 3-Aminoacrylate, and 3-Amidoiminomalonate Complexes as Catalysts for the Dimerization of Olefins
}

\author{
Martin E. Bluhm, ${ }^{\dagger}$ Cristina Folli, ${ }^{\dagger}$ Daniela Pufky,${ }^{\dagger}$ Mario Kröger ${ }^{\dagger}$ Olaf Walter, ${ }^{\dagger}$ \\ Manfred Döring* ${ }^{\dagger}$ \\ Forschungszentrum Karlsruhe, Institute for Technical Chemistry (ITC-CPV), P.O. Box 3640, \\ 76021 Karlsruhe, Germany
}

\section{Supporting Information:}

General Considerations. NMR spectra were recorded on a Bruker spectrometer $250 \mathrm{MHz}\left({ }^{1} \mathrm{H}\right)$ and 62.9 MHz $\left({ }^{13} \mathrm{C}\right)$ at $293 \mathrm{~K}$. Mass spectra were obtained using electron ionisation (EI), electron spray ionisation (ESI) or field ionisation (FI). FI- and EI-spectra were recorded with Micromass GCT and Finnigan MAT GCQ spectrometers, ESI-spectra were recorded with a Helwett-Packard 1100 MSD spectrometer. Melting points were either determined by using capillaries and an apparatus of Büchi, or with differential scanning calorimetry (DSC) with a Mettler Toledo DSC822e. IR spectra were recorded with a Perkin Elmer System 2000 FT-IR.

\section{Synthesis of ligands}

2-Cyano-N-(2,6-diisopropylphenyl)acetamide (I): Cyanoacetic acid (12.76 g, $0.15 \mathrm{~mol})$ was added to a stirred suspension of phosphorus pentachloride $(31.27 \mathrm{~g}, 0.15 \mathrm{~mol})$ in dichloromethane $(450 \mathrm{ml})$ at ambient temperature. The reaction mixture was heated at reflux for $30 \mathrm{~min}$. After cooling, 2,6-diisopropylaniline $(17.73 \mathrm{~g}, 0.10 \mathrm{~mol})$ was added in $10 \mathrm{~min}$ and the reaction mixture was heated $2 \mathrm{~h}$ under reflux. It was then cooled in an ice/water bath, and water (200 $\mathrm{ml}$ ) was added. After stirring for $30 \mathrm{~min}$, the reaction mixture was neutralized by addition of a sodium carbonate solution. The water phase was extracted twice with dichloromethane; the 
organic phases were then collected and dried over $\mathrm{Na}_{2} \mathrm{SO}_{4}$. The solvent was removed under reduced pressure and the obtained white solid was recrystallized from toluene and dried in vacuum. Yield: $23.3 \mathrm{~g}(0.095 \mathrm{~mol}, 95 \%) .{ }^{1} \mathrm{H} \mathrm{NMR}\left(\mathrm{CDCl}_{3}\right) \delta 7.73(\mathrm{~s}, 1 \mathrm{H}, \mathrm{NH}), 7.28-7.07$ (m, $\left.3 \mathrm{H}, H_{\text {Aryl }}\right), 3.33\left(\mathrm{~s}, \mathrm{CH}_{2}\right), 2.87\left(\mathrm{~m}_{7}, 2 \mathrm{H}, \mathrm{CH}\right), 1.07\left(\mathrm{~d}, 12 \mathrm{H}, \mathrm{CH}_{3}\right) . \mathrm{EI}^{+}-\mathrm{MS} m / z=244\left(\mathrm{M}^{+}\right)$. M.p. $160-162{ }^{\circ} \mathrm{C}$.

Ethyl-2-cyano-N-(2,6-diisopropylphenyl)ethanimidoate (II): A mixture of 2-cyano- $\mathrm{N}-(2,6-$ diisopropylphenyl)acetamide (I) $(14.70 \mathrm{~g}, 60 \mathrm{mmol})$ and a $1.0 \mathrm{M}$ solution of triethyloxonium tetrafluoroborate in dichloromethane $(66 \mathrm{ml}, 66 \mathrm{mmol})$ was stirred $5 \mathrm{~d}$ at ambient temperature. The solvent was removed in vacuum and the residue was washed twice with abs. diethylether. Then the residue was taken up in $60 \mathrm{ml}$ of abs. diethylether and cooled to $0{ }^{\circ} \mathrm{C}$. Triethylamine $(9.2 \mathrm{ml}, 66 \mathrm{mmol})$ was slowly added, and the mixture was stirred at r. t. for $2 \mathrm{~h}$. The organic phase was separated and the residue was washed three times with abs. diethylether. The diethylether solutions were collected, dried over $\mathrm{Na}_{2} \mathrm{SO}_{4}$, and the solvent was removed under reduced pressure. Distillation of the residue $(13.7 \mathrm{~g})$ gave the pure product. Yield: $7.52 \mathrm{~g}$ $(28 \mathrm{mmol}, 46 \%) .{ }^{1} \mathrm{H}$ NMR (DMSO-d $\left.{ }_{6}\right) \delta 7.18-6.97\left(\mathrm{~m}, 3 \mathrm{H}, H_{\text {Aryl }}\right), 4.35\left(\mathrm{q}, 2 \mathrm{H}, \mathrm{OCH}_{2}\right), 3.40(\mathrm{~s}$, $\left.2 \mathrm{H}, \mathrm{CH}_{2}\right), 2.72\left(\mathrm{~m}_{7}, 2 \mathrm{H}, \mathrm{CH}\right), 1.34\left(\mathrm{t}, 3 \mathrm{H}, \mathrm{CH}_{3}\right), 1.14\left(\mathrm{~d}, 6 \mathrm{H}, \mathrm{CH}_{3}\right), 1.06\left(\mathrm{~d}, 6 \mathrm{H}, \mathrm{CH}_{3}\right) .{ }^{13} \mathrm{C} \mathrm{NMR}$ $\left(\right.$ DMSO$\left._{-} \mathrm{d}_{6}\right) \delta 151.84,142.00,137.54,124.24,123.40,115.21,62.87,27.98,23.52,22.78,19.57$ 14.30. $\mathrm{EI}^{+}-\mathrm{MS} m / z=273\left(\mathrm{M}^{+}\right)$. B.p. $103{ }^{\circ} \mathrm{C}$.

Ethyl-2-cyano-3-ethoxy-N-(2,6-diisopropylphenyl)prop-2-enimidoate (III): A mixture of ethyl-2-cyano- $N$-(2,6-diisopropylphenyl)ethanimidoate $\quad$ (II) $\quad(7.3 \mathrm{~g}, \quad 26 \mathrm{mmol}), \quad$ triethyl orthoformate $(3.8 \mathrm{~g}, 26 \mathrm{mmol})$, and acetic anhydride $(5.47 \mathrm{~g}, 54 \mathrm{mmol})$ was refluxed for $5.5 \mathrm{~h}$. The solvent was removed under reduced pressure and the residual thick oil was distilled. Yield: $5.4 \mathrm{~g}(16 \mathrm{mmol}, 64 \%) .{ }^{1} \mathrm{H}$ NMR (DMSO-d 6$) \delta 7.88(\mathrm{~s}, 1 \mathrm{H}, \mathrm{C}=\mathrm{CH}), 7.13-6.97\left(\mathrm{~m}, 3 \mathrm{H}, H_{\text {Aryl }}\right)$, 4.29 (q, 2H, $\left.\mathrm{CH}_{2}\right), 4.21\left(\mathrm{q}, 2 \mathrm{H}, \mathrm{CH}_{2}\right), 2.80\left(\mathrm{~m}_{7}, 2 \mathrm{H}, \mathrm{CH}\right), 1.33\left(\mathrm{t}, 3 \mathrm{H}, \mathrm{CH}_{3}\right), 1.15-1.05(\mathrm{~m}, 15 \mathrm{H}$, $\left.\mathrm{CH}_{3}\right) .{ }^{13} \mathrm{C}$ NMR $\left(\mathrm{DMSO}_{-}\right) \delta$ 171.6, 149.5, 143.4, 137.4, 123.9, 123.0, 112.1, 84.1, 72.9, 62.2, 28.1, 23.2, 15.3, $14.5 . \mathrm{EI}^{+}-\mathrm{MS} m / z=329\left(\mathrm{M}^{+}\right)$. B.p. $125-130^{\circ} \mathrm{C}$. 
Ethyl-2-cyano-3-[(2,6-diisopropylphenyl)amino]-N-(2,6-diisopropylphenyl)prop-2-

enimidoate (IVa): A mixture of (III) $(1.51 \mathrm{~g}, 4.6 \mathrm{mmol})$ and 2,6-diisopropylaniline (0.93 g, $4.7 \mathrm{mmol}$ ) in $10 \mathrm{ml}$ of methanol was refluxed for $30 \mathrm{~min}$. The reaction mixture was then stored overnight at $5{ }^{\circ} \mathrm{C}$ and the product crystallized. The colorless crystals were filtered and washed with hexane. Yield: $1.21 \mathrm{~g}(2.6 \mathrm{mmol}, 57 \%) .{ }^{1} \mathrm{H}$ NMR $\left(\mathrm{CDCl}_{3}\right) \delta 7.53(\mathrm{~d}, 1 \mathrm{H}, \mathrm{C}=\mathrm{CH}), 7.28-7.02$ $\left(\mathrm{m}, 6 \mathrm{H}, H_{\text {Aryl }}\right), 4.37(\mathrm{~d}, 1 \mathrm{H}, \mathrm{NH}), 3.72\left(\mathrm{q}, 2 \mathrm{H}, \mathrm{CH}_{2}\right), 3.04\left(\mathrm{~m}_{7}, 2 \mathrm{H}, \mathrm{CH}\right), 2.89\left(\mathrm{~m}_{7}, 2 \mathrm{H}, \mathrm{CH}\right), 1.18$ $\left(\mathrm{t}, 3 \mathrm{H}, \mathrm{CH}_{3}\right), 1.13\left(\mathrm{~d}, 6 \mathrm{H}, \mathrm{CH}_{3}\right), 1.10\left(\mathrm{~d}, 6 \mathrm{H}, \mathrm{CH}_{3}\right) .{ }^{13} \mathrm{C} \mathrm{NMR}\left(\mathrm{CDCl}_{3}\right) \delta 156.8,145.4,145.0$ 139.0, 135.9, 128.7, 124.4, 124.2, 123.3, 29.1, 28.8, 28.7, 24.3, 24.0, 23.9, 23.3, 22.9, 15.6. IR $(\mathrm{KBr}): \vee 3185 \mathrm{~cm}^{-1}(\mathrm{~N}-\mathrm{H}), 2198(\mathrm{C} \equiv \mathrm{N}), 1647(\mathrm{C}=\mathrm{N}), 1587(\mathrm{C}=\mathrm{C}) . \mathrm{EI}^{+}-\mathrm{HR}-\mathrm{MS}: \mathrm{m} / z$ 459.3250 (calcd.), found 459.3255 for $\mathrm{C}_{30} \mathrm{H}_{41} \mathrm{~N}_{3} \mathrm{O}, \delta=0.5 \mathrm{mDa}$. M.p. $157{ }^{\circ} \mathrm{C}$.

Ethyl-2-cyano-3-[(2,6-dimethylphenyl)amino]-N-(2,6-diisopropylphenyl)prop-2-enimidoate

(IVb): A mixture of (III) $(0.66 \mathrm{~g}, 2.0 \mathrm{mmol})$ and 2,6-dimethylaniline $(0.25 \mathrm{~g}, 2.1 \mathrm{mmol})$ was dissolved in $7 \mathrm{ml}$ of methanol and refluxed for $30 \mathrm{~min}$. The solution was then stored overnight at $-20{ }^{\circ} \mathrm{C}$ and the product crystallized. The crystals formed were filtered and washed with hexane. Yield: $0.48 \mathrm{~g}(1.2 \mathrm{mmol}, 60 \%) .{ }^{1} \mathrm{H}$ NMR $\left(\mathrm{CDCl}_{3}\right) \delta 7.45(\mathrm{~d}, 1 \mathrm{H}, \mathrm{C}=\mathrm{CH}), 7.06-6.95(\mathrm{~m}, 6 \mathrm{H}$, $\left.H_{\text {Aryl }}\right), 4.28\left(\mathrm{q}, 2 \mathrm{H}, \mathrm{CH}_{2}\right), 2.96\left(\mathrm{~m}_{7}, 1 \mathrm{H}, \mathrm{CH}\right), 2.87\left(\mathrm{~m}_{7}, 1 \mathrm{H}, \mathrm{CH}\right), 2.19\left(\mathrm{~s}, 3 \mathrm{H}, \mathrm{CH}_{3}\right), 2.08(\mathrm{~s}, 3 \mathrm{H}$, $\left.\mathrm{CH}_{3}\right), 1.30\left(\mathrm{t}, 3 \mathrm{H}, \mathrm{CH}_{3}\right), 1.18\left(\mathrm{~d}, 3 \mathrm{H}, \mathrm{CH}_{3}\right), 1.12\left(\mathrm{~d}, 3 \mathrm{H}, \mathrm{CH}_{3}\right), 1.10\left(\mathrm{~d}, 3 \mathrm{H}, \mathrm{CH}_{3}\right), 1.07(\mathrm{~d}, 3 \mathrm{H}$, $\left.\mathrm{CH}_{3}\right) .{ }^{13} \mathrm{C} \mathrm{NMR}\left(\mathrm{CDCl}_{3}\right) \delta 155.8,150.9,142.8,141.4,133.8,133.4,129.2,127.8,127.5,124.4$, 124.0, 123.3, 115.1, 75.6, 62.2, 29.0, 28.6, 24.3, 23.8, 23.3, 22.9, 18.8, 18.6, 14.9. IR (KBr): $v$ $3185 \mathrm{~cm}^{-1}(\mathrm{~N}-\mathrm{H}), 2204(\mathrm{C} \equiv \mathrm{N}), 1619(\mathrm{C}=\mathrm{N}), 1589(\mathrm{C}=\mathrm{C}) . \mathrm{EI}^{+}-\mathrm{HR}-\mathrm{MS}: \mathrm{m} / z 403.2624$ (calcd.), found 403.2656 for $\mathrm{C}_{30} \mathrm{H}_{41} \mathrm{~N}_{3} \mathrm{O}, \delta=3.2 \mathrm{mDa}$. M.p. $195^{\circ} \mathrm{C}$.

Ethyl-2-cyano-3-[(4-dimethoxyphenyl)amino]acrylate (Va): A mixture of ethyl (ethoxymethylene)cyanoacetate $(2.80 \mathrm{~g}, 16 \mathrm{mmol})$ and $p$-anisidine $(2.05 \mathrm{~g}, 16 \mathrm{mmol})$ in $10 \mathrm{ml}$ of methanol was refluxed for $45 \mathrm{~min}$. The obtained solution was cooled to r. t. and a pale grey solid precipitated, which was filtered and washed with hexane. Yield: $3.84 \mathrm{~g}$ (15 mmol, 96\%). ${ }^{1} \mathrm{H}$ NMR $\left(\mathrm{CDCl}_{3}\right) \delta 10.69(\mathrm{~d}, 1 \mathrm{H}, \mathrm{NH}), 7.75(\mathrm{~d}, 1 \mathrm{H}, \mathrm{N}-\mathrm{CH}), 7.03\left(\mathrm{~d}, 2 \mathrm{H}, H_{\text {Aryl }}\right), 6.91(\mathrm{~d}, 2 \mathrm{H}$, $\left.H_{\text {Aryl }}\right), 4.28\left(\mathrm{q}, 2 \mathrm{H}, \mathrm{CH}_{2}\right), 3.81\left(\mathrm{~s}, 3 \mathrm{H}, \mathrm{OCH}_{3}\right), 1.35\left(\mathrm{t}, 3 \mathrm{H}, \mathrm{CH}_{3}\right) .{ }^{13} \mathrm{C} \mathrm{NMR}\left(\mathrm{CDCl}_{3}\right) \delta 168.1$, 
158.1, 152.8, 132.2, 119.2, 118.6, 115.5, 74.6, 61.4, 55.9, 14.6. $\mathrm{FI}^{+}-\mathrm{HR}-\mathrm{MS}: m / z$ 246.1004 (calcd.), found 246.0987 for $\mathrm{C}_{13} \mathrm{H}_{14} \mathrm{~N}_{2} \mathrm{O}_{3}, \delta=1.7 \mathrm{mDa}$. M.p. 106.1-107.1 ${ }^{\circ} \mathrm{C}$.

Ethyl-2-cyano-3-[(2,6-dimethylphenyl)amino]acrylate (Vb): 2,6-Dimethylaniline (3.72 g, $30 \mathrm{mmol}$ ) was added dropwise to a solution of ethyl(ethoxymethylene)cyanoacetate $(5.23 \mathrm{~g}$, $30 \mathrm{mmol}$ ) in $10 \mathrm{ml}$ of methanol. The mixture was refluxed for $20 \mathrm{~min}$; then it was cooled to r. $\mathrm{t}$. and small colorless crystals crystallized from the solution, which were filtered and washed with hexane. Yield: $6.11 \mathrm{~g}(25 \mathrm{mmol}, 83 \%) .{ }^{1} \mathrm{H}$ NMR $\left(\mathrm{CDCl}_{3}\right) \delta 10.17(\mathrm{~d}, 1 \mathrm{H}, \mathrm{NH}), 7.28(\mathrm{~d}, 1 \mathrm{H}, \mathrm{N}-$ $\mathrm{CH}), 7.07-7.00\left(\mathrm{~m}, 3 \mathrm{H}, \mathrm{H}_{\text {Aryl }}\right), 4.16\left(\mathrm{q}, 2 \mathrm{H}, \mathrm{CH}_{2}\right), 2.21\left(\mathrm{~s}, 6 \mathrm{H}, \mathrm{CH}_{3}\right), 1.25\left(\mathrm{t}, 3 \mathrm{H}, \mathrm{CH}_{3}\right) .{ }^{13} \mathrm{C} \mathrm{NMR}$ $\left(\mathrm{CDCl}_{3}\right) \delta 166.3,158.9,136.9,132.9,129.0,127.8,117.0,74.1,60.8,18.3,14.4 . \mathrm{IR}(\mathrm{KBr}): v$ $3196 \mathrm{~cm}^{-1}(\mathrm{~N}-\mathrm{H}), 2213(\mathrm{C} \equiv \mathrm{N}), 1691(\mathrm{C}=\mathrm{C}-\mathrm{N}), 1599(\mathrm{~N}-\mathrm{H}) \cdot \mathrm{FI}^{+}-\mathrm{HR}-\mathrm{MS}: m / z 244.1212$ (calcd.), found 244.1160 for $\mathrm{C}_{14} \mathrm{H}_{16} \mathrm{~N}_{2} \mathrm{O}_{2}, \delta=5.2 \mathrm{mDa}$. M.p. $138.2-139.5^{\circ} \mathrm{C}$.

Ethyl-2-cyano-3-[(2,6-diethylphenyl)amino]acrylate (Vc): A mixture of ethyl(ethoxymethylene)cyanoacetate $(3.02 \mathrm{~g}, 18 \mathrm{mmol})$ and 2,6-diethylaniline $(2.69 \mathrm{~g}, 18 \mathrm{mmol})$ in $10 \mathrm{ml}$ of methanol was refluxed for $30 \mathrm{~min}$. The reaction mixture was cooled to $\mathrm{r}$. $\mathrm{t}$. and stored overnight at $+5{ }^{\circ} \mathrm{C}$ : needle-shaped colorless crystals appeared, which were filtered and washed with hexane. Yield: $4.08 \mathrm{~g}(15 \mathrm{mmol}, 86 \%) .{ }^{1} \mathrm{H}$ NMR $\left(\mathrm{CDCl}_{3}\right) \delta 10.24(\mathrm{~d}, 1 \mathrm{H}, \mathrm{NH}), 7.86(\mathrm{~d}, 1 \mathrm{H}, \mathrm{N}-$ $\mathrm{CH}), 7.34-7.13\left(\mathrm{~m}, 3 \mathrm{H}, H_{\text {Aryl }}\right), 4.26\left(\mathrm{q}, 2 \mathrm{H}, \mathrm{OCH}_{2}\right), 2.62\left(\mathrm{q}, 4 \mathrm{H}, \mathrm{CH}_{2}\right), 1.37\left(\mathrm{t}, 3 \mathrm{H}, \mathrm{CH}_{3}\right), 1.21(\mathrm{t}$, $\left.6 \mathrm{H}, \mathrm{CH}_{3}\right) .{ }^{13} \mathrm{C} \mathrm{NMR}\left(\mathrm{CDCl}_{3}\right) \delta 167.9,159.2,140.1,135.7,128.6,127.2,116.9,73.4,60.9,24.7$, 14.7, 14.3. $\mathrm{FI}^{+}$-HR-MS: $m / z 272.1525$ (calcd.), found 272.1477 for $\mathrm{C}_{16} \mathrm{H}_{20} \mathrm{~N}_{2} \mathrm{O}_{2}, \delta=4.8 \mathrm{mDa}$. M.p. $114.6-115.9^{\circ} \mathrm{C}$.

Ethyl-2-cyano-3-[(2,6-diisopropylphenyl)amino]acrylate (Vd): Ethyl(ethoxymethylene)cyanoacetate $(5.20 \mathrm{~g}, 30 \mathrm{mmol})$ was added to a solution of 2,6-diisopropylaniline (5.93 $\mathrm{g}$, $30 \mathrm{mmol}$ ) in $100 \mathrm{ml}$ of methanol. The mixture was refluxed for $10 \mathrm{~min}$; the solvent was then removed under reduced pressure, and the resulting residue was recrystallized from ethyl acetate. The colorless crystals were filtered and washed with hexane. Yield: $3.75 \mathrm{~g}(12 \mathrm{mmol}, 42 \%) .{ }^{1} \mathrm{H}$ $\operatorname{NMR}\left(\mathrm{CDCl}_{3}\right) \delta 10.15(\mathrm{~d}, 1 \mathrm{H}, \mathrm{NH}), 7.72(\mathrm{~d}, 1 \mathrm{H}, \mathrm{N}-\mathrm{CH}), 7.30-7.11\left(\mathrm{~m}, 3 \mathrm{H}, H_{\text {Aryl }}\right), 4.19(\mathrm{q}, 2 \mathrm{H}$, $\left.\mathrm{CH}_{2}\right), 2.98(\mathrm{~m}, 2 \mathrm{H}, \mathrm{CH}), 1.29\left(\mathrm{t}, 3 \mathrm{H}, \mathrm{CH}_{3}\right), 1.15\left(\mathrm{~d}, 6 \mathrm{H}, \mathrm{CH}_{3}\right) .{ }^{13} \mathrm{C} \mathrm{NMR}\left(\mathrm{CDCl}_{3}\right) \delta 166.7,159.9$, 
145.0, 134.4, 129.3, 124.5, 117.4, 74.3, 61.3, 28.8, 24.0, 14.7. IR (KBr): v $3202 \mathrm{~cm}^{-1}(\mathrm{~N}-\mathrm{H})$, $2216(\mathrm{C} \equiv \mathrm{N}), 1674(\mathrm{C}=\mathrm{C}-\mathrm{N}), 1623(\mathrm{C}=\mathrm{C}) . \mathrm{FI}^{+}-\mathrm{HR}-\mathrm{MS}: \mathrm{m} / z, 300.1838$ (calcd.), found 300.1745 for $\mathrm{C}_{18} \mathrm{H}_{24} \mathrm{~N}_{2} \mathrm{O}_{2}, \delta=9.3 \mathrm{mDa}$. M.p. $157-158{ }^{\circ} \mathrm{C}$.

Ethyl-2-cyano-3-[(4-methylphenyl)amino]acrylate (Ve): A mixture of equimolar amounts of ethyl(ethoxymethylene)cyanoacetate $(5.25 \mathrm{~g}, 30 \mathrm{mmol})$ and $p$-toluidine $(3.25 \mathrm{~g}, 30 \mathrm{mmol})$ in $30 \mathrm{ml}$ of methanol was refluxed for $15 \mathrm{~min}$. The reaction mixture was then cooled to r. t. and the volume of the solvent was reduced to $10 \mathrm{ml}$ under reduced pressure: the needle shaped colorless crystals were filtered and washed with hexane. Yield: $6.64 \mathrm{~g}(29 \mathrm{mmol}, 96 \%) .{ }^{1} \mathrm{H} \mathrm{NMR}\left(\mathrm{CDCl}_{3}\right)$ $\delta 10.63(\mathrm{~d}, 1 \mathrm{H}, \mathrm{NH}), 7.75(\mathrm{~d}, 1 \mathrm{H}, \mathrm{N}-\mathrm{CH}), 7.10\left(\mathrm{~d}, 2 \mathrm{H}, H_{\text {Aryl }}\right), 6.90\left(\mathrm{~d}, 2 \mathrm{H}, H_{\text {Aryl }}\right), 4.20(\mathrm{q}, 2 \mathrm{H}$, $\left.\mathrm{CH}_{2}\right), 2.26\left(\mathrm{~s}, 3 \mathrm{H}, \mathrm{CH}_{3}\right), 1.27\left(\mathrm{t}, 3 \mathrm{H}, \mathrm{CH}_{3}\right) .{ }^{13} \mathrm{C} \mathrm{NMR}\left(\mathrm{CDCl}_{3}\right) \delta 167.96,152.41,136.18$ 130.86, 130.77, 118.05, 117.54, 75.18, 61.43, 21.12, 14.61. FI'-HR-MS: m/z 230.1055 (calcd.), found 230.1024 for $\mathrm{C}_{13} \mathrm{H}_{14} \mathrm{~N}_{2} \mathrm{O}_{2}, \delta=3.1 \mathrm{mDa}$. M.p. $128.7-128.9^{\circ} \mathrm{C}$.

Diethyl\{[(4-methylphenyl)amino]methylene\}malonate (VIa): A mixture of diethyl ethoxymethylenemalonate $(4.06 \mathrm{~g}, 18.4 \mathrm{mmol})$ and $p$-toluidine $(1.98 \mathrm{~g}, 18.3 \mathrm{mmol})$ in $5 \mathrm{ml}$ of ethanol was heated at $140{ }^{\circ} \mathrm{C}$ for $5 \mathrm{~h}$, while the produced ethanol was distilled out of the reaction mixture. The resulting thick oil was stored overnight at $-30{ }^{\circ} \mathrm{C}$ and a solid formed, which was crushed in a mortar. The solid was suspended in $20 \mathrm{ml}$ of cold hexane and stirred for $1 \mathrm{~h}$. Finally, the white powder was filtered. Yield: $4.34 \mathrm{~g}(15.6 \mathrm{mmol}, 85 \%) .{ }^{1} \mathrm{H} \mathrm{NMR}\left(\mathrm{CDCl}_{3}\right) \delta 10.98(\mathrm{~d}$, $1 \mathrm{H}, \mathrm{NH}), 8.50(\mathrm{~d}, 1 \mathrm{H}, \mathrm{CH}), 7.17\left(\mathrm{~d}, 2 \mathrm{H}, H_{\text {Aryl }}\right), 7.03\left(\mathrm{~d}, 2 \mathrm{H}, H_{\text {Aryl }}\right), 4.30\left(\mathrm{q}, 2 \mathrm{H}, \mathrm{CH}_{2}\right), 4.24$ (q, $\left.2 \mathrm{H}, \mathrm{CH}_{2}\right), 2.33\left(\mathrm{~s}, 3 \mathrm{H}, \mathrm{CH}_{3}\right), 1.38\left(\mathrm{t}, 3 \mathrm{H}, \mathrm{CH}_{3}\right), 1.32\left(\mathrm{t}, 3 \mathrm{H}, \mathrm{CH}_{3}\right) .{ }^{13} \mathrm{C} \mathrm{NMR}\left(\mathrm{CDCl}_{3}\right) \delta 169.5$, $166.1,152.5,137.3,135.1,130.7,117.6,93.4,60.6,60.3,21.1,14.8,14.7 . \mathrm{FI}^{+}-\mathrm{HR}-\mathrm{MS}:$ $m / z 277.1314$ (calcd.), found 277.1193 for $\mathrm{C}_{15} \mathrm{H}_{19} \mathrm{NO}_{4}, \delta=12.1 \mathrm{mDa}$. M.p. $47.5-47.7^{\circ} \mathrm{C}$.

Diethyl\{[(2,6-diethylphenyl)amino]methylene $\}$ malonate (VIb): A solution of 2,6diethylaniline $(2.79 \mathrm{~g}, 18.3 \mathrm{mmol})$ in $5 \mathrm{ml}$ of ethanol was dropped into diethyl ethoxymethylenemalonate $(4.00 \mathrm{~g}, 18.1 \mathrm{mmol})$ while stirring at $\mathrm{r}$. t.. The mixture was then heated at $150^{\circ} \mathrm{C}$ for $5 \mathrm{~h}$ and the produced ethanol was distilled out of the reaction mixture. An oil remained, which was stored overnight at $-30{ }^{\circ} \mathrm{C}$ where it solidified. The solid was then crushed 
in a mortar, taken in $10 \mathrm{ml}$ of cold hexane, stirred for $30 \mathrm{~min}$, and was finally filtered. A white powder was isolated. Yield: $4.78 \mathrm{~g}(15.0 \mathrm{mmol}, 83 \%) .{ }^{1} \mathrm{H} \mathrm{NMR}\left(\mathrm{CDCl}_{3}\right) \delta 10.50(\mathrm{~d}, 1 \mathrm{H}, \mathrm{NH})$, $8.04(\mathrm{~d}, 1 \mathrm{H}, \mathrm{CH}), 7.26-7.13\left(\mathrm{~m}, 3 \mathrm{H}, H_{\text {Aryl }}\right), 4.33$ (q, 2H, $\left.\mathrm{CH}_{2}\right), 4.19$ (q, 2H, $\left.\mathrm{CH}_{2}\right), 2.64$ (q, 4H, $\left.\mathrm{CH}_{2}\right), 1.40\left(\mathrm{t}, 3 \mathrm{H}, \mathrm{CH}_{3}\right), 1.27\left(\mathrm{t}, 3 \mathrm{H}, \mathrm{CH}_{3}\right), 1.22\left(\mathrm{t}, 6 \mathrm{H}, \mathrm{CH}_{3}\right) .{ }^{13} \mathrm{C} \mathrm{NMR}\left(\mathrm{CDCl}_{3}\right) \delta$ 169.6, 166.0, 160.0, 139.8, 137.2, 128.0, 127.5, 91.9, 60.4, 60.0, 25.2, 15.1, 14.7. FI ${ }^{+}-\mathrm{HR}-\mathrm{MS}: \mathrm{m} / \mathrm{z} 319.1784$ (calcd.), found 319.1744 for $\mathrm{C}_{18} \mathrm{H}_{25} \mathrm{NO}_{4}, \delta=4.0 \mathrm{mDa}$. M.p. $77.4-78.4^{\circ} \mathrm{C}$.

Diethyl\{[(2,6-diisopropylphenyl)amino]methylene \}malonate (VIc): A mixture of diethyl ethoxymethylenemalonate $(3.31 \mathrm{~g}, 15 \mathrm{mmol})$ and 2,6-diisopropylaniline $(2.96 \mathrm{~g}, 15 \mathrm{mmol})$ was heated at $150{ }^{\circ} \mathrm{C}$ for $4 \mathrm{~h}$ while the produced ethanol was distilled out of the reaction mixture. An oil resulted, which was stored overnight at $-30^{\circ} \mathrm{C}$; the formed solid was crushed in a mortar, then mixed with $10 \mathrm{ml}$ of cold hexane, and stirred for $30 \mathrm{~min}$. The white powder was finally filtered. Yield: $3.73 \mathrm{~g}(10.7 \mathrm{mmol}, 71 \%) .{ }^{1} \mathrm{H} \mathrm{NMR}\left(\mathrm{CDCl}_{3}\right) \delta 10.41(\mathrm{~d}, 1 \mathrm{H}, \mathrm{NH}), 7.92(\mathrm{~d}, 1 \mathrm{H}$, $\mathrm{CH}), 7.27-7.11\left(\mathrm{~m}, 3 \mathrm{H}, \mathrm{H}_{\text {Aryl }}\right), 4.26\left(\mathrm{q}, 2 \mathrm{H}, \mathrm{CH}_{2}\right), 4.11\left(\mathrm{q}, 2 \mathrm{H}, \mathrm{CH}_{2}\right), 3.04(\mathrm{~m}, 2 \mathrm{H}, \mathrm{CH}), 1.33(\mathrm{t}$, $\left.3 \mathrm{H}, \mathrm{CH}_{3}\right), 1.18\left(\mathrm{t}, 3 \mathrm{H}, \mathrm{CH}_{3}\right), 1.16\left(\mathrm{~d}, 12 \mathrm{H}, \mathrm{CH}_{3}\right) .{ }^{13} \mathrm{C} \mathrm{NMR}\left(\mathrm{CDCl}_{3}\right) \delta 169.4,160.2,144.3,135.6$, 128.2, 124.0, 91.4, 60.1, 28.4, 23.7, 14.4. FI'-HR-MS: $m / z$ 347.2097 (calcd.), found 347.2111 for $\mathrm{C}_{20} \mathrm{H}_{29} \mathrm{NO}_{4}, \delta=1.4 \mathrm{mDa}$. M.p. $77.0-77.8^{\circ} \mathrm{C}$.

Ethyl-3-[(2,6-dimethylphenyl)amino]-3-oxopropanoate (VId): A mixture of diethyl malonate (48.0 g, $0.30 \mathrm{~mol})$ and 2,6-dimethylaniline $(12.01 \mathrm{~g}, 0.10 \mathrm{mmol})$ was heated at $160{ }^{\circ} \mathrm{C}$ for $20 \mathrm{~h}$. The produced ethanol was distilled out of the reaction mixture. Then the mixture was cooled to r. t., mixed with $80 \mathrm{ml}$ of cold hexane, stirred in an ice-bath for $1 \mathrm{~h}$, and the white solid was filtered. Yield: $22.14 \mathrm{~g}(0.09 \mathrm{~g}, 94 \%) .{ }^{1} \mathrm{H} \mathrm{NMR}\left(\mathrm{CDCl}_{3}\right) \delta 8.48(\mathrm{~s}, 1 \mathrm{H}, \mathrm{NH}), 7.07-6.98(\mathrm{~m}, 3 \mathrm{H}$, $\left.H_{\text {Aryl }}\right), 4.20\left(\mathrm{q}, 2 \mathrm{H}, \mathrm{OCH}_{2}\right), 3.44\left(\mathrm{~s}, 2 \mathrm{H}, \mathrm{CH}_{2} \mathrm{C}=\mathrm{O}\right), 2.16\left(\mathrm{~s}, 6 \mathrm{H}, \mathrm{CH}_{3}\right), 1.26\left(\mathrm{t}, 3 \mathrm{H}, \mathrm{CH}_{3}\right)$. ${ }^{13} \mathrm{C} \mathrm{NMR}\left(\mathrm{CDCl}_{3}\right) \delta 169.6,163.5,135.2,133.6,128.1,127.3,61.4,41.4,18.3,14.1 . \mathrm{EI}^{+}-\mathrm{HR}-$ MS: $m / z$ 235.1208 (calcd.), found 235.1200 for $\mathrm{C}_{13} \mathrm{H}_{17} \mathrm{NO}_{3}, \delta=0.8 \mathrm{mDa}$. M.p. 88.7-89.9 ${ }^{\circ} \mathrm{C}$.

Ethyl-3-[(2,6-diisopropylphenyl)amino]-3-oxopropanoate (VIe): A mixture of 2,6diisopropylaniline (15 g, $76 \mathrm{mmol})$ and diethyl malonate $(50.00 \mathrm{~g}, 309 \mathrm{mmol})$ was heated at 160 ${ }^{\circ} \mathrm{C}$ for $19 \mathrm{~h}$ while the produced ethanol was distilled out of the reaction mixture. A thick oil 
resulted, which was then mixed with $170 \mathrm{ml}$ of cold hexane, stirred for $1 \mathrm{~h}$, and finally filtered. The operation was repeated with further $150 \mathrm{ml}$ of hexane and a white powder recrystallized in ethanol:water $=3: 1$. Yield: $13.19 \mathrm{~g}(45 \mathrm{mmol}, 60 \%) .{ }^{1} \mathrm{H} \mathrm{NMR}\left(\mathrm{CDCl}_{3}\right) \delta 8.53(\mathrm{~s}, 1 \mathrm{H}, \mathrm{NH}), 7.31$ $\left(\mathrm{t}, 1 \mathrm{H}, H_{\text {Aryl }}\right), 7.19\left(\mathrm{~d}, 2 \mathrm{H}, H_{\text {Aryl }}\right), 4.28\left(\mathrm{q}, 2 \mathrm{H}, \mathrm{OCH}_{2}\right), 3.53\left(\mathrm{~s}, 2 \mathrm{H}, \mathrm{CH}_{2} \mathrm{C}=\mathrm{O}\right), 3.05\left(\mathrm{~m}_{7}, 2 \mathrm{H}, \mathrm{CH}\right)$, $1.35\left(\mathrm{t}, 3 \mathrm{H}, \mathrm{CH}_{3}\right), 1.20\left(\mathrm{~d}, 12 \mathrm{H}, \mathrm{CH}_{3}\right) .{ }^{13} \mathrm{C} \mathrm{NMR}\left(\mathrm{CDCl}_{3}\right) \delta 169.9,164.4,146.0,130.9,128.4$, 123.5, 61.8, 41.2, 28.8, 23.6, 14.1. EI ${ }^{+}-\mathrm{HR}-\mathrm{MS}: \mathrm{m} / \mathrm{z} 291.1834$ (calcd.), found 291.1788 for $\mathrm{C}_{17} \mathrm{H}_{25} \mathrm{NO}_{3}, \delta=4.6 \mathrm{mDa}$. M.p. $127.2-127.9^{\circ} \mathrm{C}$.

Ethyl-2-cyano-3-[(1-pyridine-2-ylethyl)amino]acrylate $\quad$ (VIIc): $\quad$ 2-cyano-3-ethoxyethylacrylate $(1.68 \mathrm{~g}, 9.94 \mathrm{mmol})$ and 1-pyridine-2-ylethanamine ${ }^{[1]}(1.21 \mathrm{~g} ; 9.94 \mathrm{mmol})$ were dissolved in $7 \mathrm{ml}$ of methanol and heated under reflux for $30 \mathrm{~min}$. Then, the solvent was removed and the residue dried in vacuum. The oily product was immediately used in the next reaction step to give VIIIc. Yield: $2.41 \mathrm{~g}(9.83 \mathrm{mmol}, 99 \%) .{ }^{1} \mathrm{H} \mathrm{NMR}\left(\mathrm{CHCl}_{3}\right) \delta 9.61$ (bs, $1 \mathrm{H}$, $\mathrm{NH}) ; 8.55(\mathrm{~m}, 1 \mathrm{H}) ; 7.97(\mathrm{~d}, \mathrm{CH}=\mathrm{C}) ; 7.64(\mathrm{~m}, 1 \mathrm{H}) ; 7.38(\mathrm{~d}, \mathrm{CH}=\mathrm{C}) ; 7.16(\mathrm{~m}, 2 \mathrm{H}) ; 4.59(\mathrm{q}, 1 \mathrm{H}$, $\left.\mathrm{CH}-\mathrm{CH}_{3}\right) ; 4.16\left(\mathrm{q}, 2 \mathrm{H}, \mathrm{CH}_{2}-\mathrm{CH}_{3}\right) ; 1.56$ (d, 3H, $\left.\mathrm{CH}_{3}-\mathrm{CH}\right) ; 4.59$ (t, 3H, $\mathrm{CH}_{3}-\mathrm{CH}_{2}$ ). EI ${ }^{+}-\mathrm{HR}-\mathrm{MS}$ : $m / z=245.1164$ (calcd.), found 245.1165 for $\mathrm{C}_{13} \mathrm{H}_{15} \mathrm{~N}_{3} \mathrm{O}_{2}, \delta=0.1 \mathrm{mDa}$.

Ethyl-2-acetyl-3-[(2,6-dimethylphenyl)amino]acrylate (VIIIa): A mixture of ethyl acetoacetate $(4.87 \mathrm{~g}, 37 \mathrm{mmol})$, triethyl orthoformate $(10.31 \mathrm{~g}, 68 \mathrm{mmol})$ and acetic anhydride $(3.96 \mathrm{~g}, 38 \mathrm{mmol})$ was refluxed for $70 \mathrm{~min}$ and then the volatile components were removed in vacuum (typically $60^{\circ} \mathrm{C} / 0.2 \mathrm{mmHg}$ ). The residual liquid consists largely of ethyl-2-acetyl-3ethoxyacrylate (VIIa) and was used without purification. It was heated to $150^{\circ} \mathrm{C}$ and hot 2,6dimethylaniline $(9.00 \mathrm{~g}, 74 \mathrm{mmol})$ was quickly added. The mixture was refluxed for $20 \mathrm{~min}$, then it was cooled and $50 \mathrm{ml}$ of cold hexane were added under vigorously stirring. The mixture was stored overnight at $-30{ }^{\circ} \mathrm{C}$. Colorless crystals formed, which were filtered and washed with hexane. Yield: $4.28 \mathrm{~g}(16 \mathrm{mmol}, 44 \%) .{ }^{1} \mathrm{H}$ NMR $\left(\mathrm{CDCl}_{3}\right) \delta 12.31(\mathrm{~d}, 1 \mathrm{H}, \mathrm{NH}), 8.08(\mathrm{~d}, 1 \mathrm{H}$, $\mathrm{C}=\mathrm{CH}), 7.12\left(\mathrm{bs}, 3 \mathrm{H}, H_{\text {Aryl }}\right), 4.20\left(\mathrm{q}, 2 \mathrm{H}, \mathrm{OCH}_{2}\right), 2.57\left(\mathrm{~s}, 3 \mathrm{H}, \mathrm{C}(\mathrm{O}) \mathrm{CH}_{3}\right), 2.31\left(\mathrm{~s}, 6 \mathrm{H}, \mathrm{CH}_{3}\right), 1.29$ $\left(\mathrm{t}, 3 \mathrm{H}, \mathrm{CH}_{3}\right) .{ }^{13} \mathrm{C} \mathrm{NMR}\left(\mathrm{CDCl}_{3}\right) \delta 200.5,167.3,159.5,138.1,132.8,129.3,127.5,101.6,60.0$, 
31.4, 18.8, 14.8. $\mathrm{EI}^{+}-\mathrm{HR}-\mathrm{MS}: \mathrm{m} / z 261.1365$ (calcd.), found 261.1351 for $\mathrm{C}_{15} \mathrm{H}_{19} \mathrm{NO}_{3}, \delta=-$ $1.4 \mathrm{mDa}$ M.p. $56 \cdot 0-56.7^{\circ} \mathrm{C}$.

Ethyl-2-butyryl-3-[(2,6-dimethylphenyl)amino]acrylate (VIIIb): A mixture of ethyl butyrylacetate $(3.01 \mathrm{~g}, 18 \mathrm{mmol})$, triethyl orthoformate $(5.44 \mathrm{~g}, 36 \mathrm{mmol})$ and acetic anhydride $(1.86 \mathrm{~g}, 18 \mathrm{mmol})$ was refluxed for $5.5 \mathrm{~h}$. Then the volatile components were removed in vacuum $\left(60^{\circ} \mathrm{C} / 0.2\right.$ torr). The residual liquid largely consisted of ethyl-2-butyryl-3-ethoxyacrylate (VIIb) and was used without purification. It was heated to $150{ }^{\circ} \mathrm{C}$ and 2,6 -dimethylaniline $(2.30 \mathrm{~g}, 26 \mathrm{mmol})$ was quickly added. The mixture was refluxed for $25 \mathrm{~min}$, then it was cooled and stored overnight at $-30{ }^{\circ} \mathrm{C}$ where it solidified. The solid was crushed, mixed with $20 \mathrm{ml}$ of cold hexane, and then stirred $15 \mathrm{~min}$ in an ice-bath. The white solid was filtered and washed with hexane. Yield: $0.74 \mathrm{~g}(3 \mathrm{mmol}, 14 \%) .{ }^{1} \mathrm{H}$ NMR $\left(\mathrm{CDCl}_{3}\right) \delta 12.31(\mathrm{~d}, 1 \mathrm{H}, \mathrm{NH}), 8.08(\mathrm{~d}, 1 \mathrm{H}$, $\mathrm{C}=\mathrm{CH}), 7.12\left(\mathrm{sb}, 3 \mathrm{H}, H_{\text {Aryl }}\right), 4.20\left(\mathrm{q}, 2 \mathrm{H}, \mathrm{OCH}_{2}\right), 2.96\left(\mathrm{t}, 2 \mathrm{H}, \mathrm{C}(\mathrm{O}) \mathrm{CH}_{2}\right), 2.31\left(\mathrm{~s}, 6 \mathrm{H}, \mathrm{CH}_{3}\right), 1.70$ $\left(\mathrm{m}_{6}, 2 \mathrm{H}, \mathrm{CH}_{2}\right), 1.29\left(\mathrm{t}, 3 \mathrm{H}, \mathrm{CH}_{3}\right), 1.00\left(\mathrm{t}, 3 \mathrm{H}, \mathrm{CH}_{3}\right) .{ }^{13} \mathrm{C} \mathrm{NMR}\left(\mathrm{CDCl}_{3}\right) \delta 202.8,167.0,159.2$, 137.8, 132.5, 128.9, 127.0, 101.1, 59.6, 44.2, 18.5, 18.4, 14.5, 14.1. EI ${ }^{+}-\mathrm{HR}-\mathrm{MS}: \mathrm{m} / z$ 289.1678 (calcd), found 289.1703 for $\mathrm{C}_{17} \mathrm{H}_{23} \mathrm{NO}_{3}, \delta=2.5 \mathrm{mDa}$. M.p. $63.5-64.2^{\circ} \mathrm{C}$.

Ethyl-(2E)-cyano(1-methylimidazo[1,5-a]pyridine-3(2H)-ylidene)acetate (VIIIc): Under argon, a mixture of VIIc $(1.56 \mathrm{~g}, 6.36 \mathrm{mmol})$ and $\mathrm{CuCl}_{2}(1.71 \mathrm{~g}, 12.72 \mathrm{mmol})$ in $20 \mathrm{ml}$ of abs. methanol was heated to $85^{\circ} \mathrm{C}$. Triethylamine $(1.29 \mathrm{~g}, 12.72 \mathrm{mmol})$ was added dropwise, and the solution was heated under reflux for $18 \mathrm{~h}$. After cooling to ambient temperature and removing the solvent at reduced pressure, a solution containing $20 \mathrm{ml}$ of water and $10 \mathrm{ml}$ of concentrated ammonia solution was added, and the mixture was stirred for $30 \mathrm{~min}$ at $40{ }^{\circ} \mathrm{C}$ under air. The formed precipitate was collected by filtration and washed with distilled water. The fluorescent product was isolated by column chromatography on silica (chloroform:ethylacetate $=2: 1$ ) and recrystallized from hexane:ethylacetate $=3: 1$. Yield: $0.20 \mathrm{~g}(0.82 \mathrm{mmol}, 13 \%) .{ }^{1} \mathrm{H}$ NMR $\left(\mathrm{CHCl}_{3}\right) \delta 12.36(\mathrm{~s}, 1 \mathrm{H}, \mathrm{NH}), 8.51(\mathrm{~d}, 1 \mathrm{H}), 7.00(\mathrm{~d}, 1 \mathrm{H}), 6.33(\mathrm{~m}, 1 \mathrm{H}), 6.51(\mathrm{~m}, 1 \mathrm{H}), 7.18(\mathrm{q}, 2 \mathrm{H}$ $\left.\mathrm{CH}_{2} \mathrm{CH}_{3}\right), 2.35\left(\mathrm{~s}, 3 \mathrm{H}, \mathrm{CH}_{3}\right), 7.18\left(\mathrm{q}, 3 \mathrm{H}, \mathrm{CH}_{3} \mathrm{CH}_{2}\right) .{ }^{13} \mathrm{C} \mathrm{NMR}\left(\mathrm{CHCl}_{3}\right) \delta 171.05(\mathrm{C}=\mathrm{O}), 138.94 ;$ $123.52,123.08,121.63,120.26,117.94,113.26,112.26,60.39\left(C_{2} \mathrm{CH}_{3}\right), 50.18\left(C_{\mathrm{sp} 3}\right), 15.08$ 
$\left(\mathrm{CH}_{3}\right), 9.64\left(\mathrm{CH}_{3} \mathrm{CH}_{2}\right) . \mathrm{EI}^{+}$-HR-MS: $\mathrm{m} / z=243.1008$ (calcd.), found 243.1047 for $\mathrm{C}_{13} \mathrm{H}_{13} \mathrm{~N}_{3} \mathrm{O}_{2} \delta$ $=3.9 \mathrm{mDa}$. M.p. $155.3^{\circ} \mathrm{C}$.

Ethyl-3-[(2,6-dimethylphenyl)amino]-2-\{[(2,6-dimethylphenyl)amino]carbonyl\}acrylate

(IXa): A mixture of diethyl ethoxymethylenemalonate $(3.00 \mathrm{~g}, 13.6 \mathrm{mmol})$ and 2,6dimethylaniline (3.35 g, $27.4 \mathrm{mmol}$ ) was heated at $135{ }^{\circ} \mathrm{C}$ for $20 \mathrm{~h}$ while the produced ethanol was distilled out of the reaction mixture. The mixture was then cooled and $70 \mathrm{ml}$ of cold hexane were added; the mixture was stirred in an ice-bath for $1 \mathrm{~h}$, then the white precipitate was filtered and washed with hexane. Colorless crystals were obtained by recrystallisation in hexane. Yield: $4.32 \mathrm{~g}(11.8 \mathrm{mmol}, 87 \%) .{ }^{1} \mathrm{H}$ NMR $\left(\mathrm{CDCl}_{3}\right) \delta 11.80(\mathrm{~d}, 1 \mathrm{H}, \mathrm{NH}), 10.22(\mathrm{~s}, 1 \mathrm{H}, \mathrm{NH}), 8.16(\mathrm{~d}$, 1H, NCH), 7.10 (bs, 6H, $\left.H_{\text {Aryl }}\right), 4.25$ (q, 2H, $\mathrm{CH}_{2}$ ), 2.33 (s, 6H, $\left.\mathrm{CH}_{3}\right), 2.32$ (s, 6H, $\left.\mathrm{CH}_{3}\right), 1.32$ (t, $\left.3 \mathrm{H}, \mathrm{CH}_{3}\right) .{ }^{13} \mathrm{C} \mathrm{NMR}\left(\mathrm{CDCl}_{3}\right) \delta 169.1,168.0,158.5,138.6,135.8,132.9,129.3,128.4,127.0$, 126.9, 100.0, 91.2, 60.4, 19.1, 19.0, 14.8. $\mathrm{EI}^{+}-\mathrm{HR}-\mathrm{MS}: \mathrm{m} / \mathrm{z} 366.1943$ (calcd.), found 366.1926 for $\mathrm{C}_{22} \mathrm{H}_{26} \mathrm{~N}_{2} \mathrm{O}_{4}, \delta=1.7 \mathrm{mDa}$. M.p. $125.1-125.7^{\circ} \mathrm{C}$.

Ethyl-3-[(2,6-diisopropylphenyl)amino]-2-\{[(2,6-diisopropylphenyl)amino]carbonyl\}acrylate (IXb): A mixture of diethyl ethoxymethylenemalonate $(5.01 \mathrm{~g}, 23 \mathrm{mmol})$ and 2,6diisopropylaniline $(9.04 \mathrm{~g}, 46 \mathrm{mmol})$ was heated at $135{ }^{\circ} \mathrm{C}$ for $38 \mathrm{~h}$; the produced ethanol was distilled out of the reaction mixture. The mixture was cooled and $20 \mathrm{ml}$ of cold hexane were added under vigorously stirring. The solution was stored over night at $-30{ }^{\circ} \mathrm{C}$ and a white precipitate was collected and filtered. Yield: $7.43 \mathrm{~g}(16 \mathrm{mmol}, 68 \%) .{ }^{1} \mathrm{H} \mathrm{NMR}\left(\mathrm{CDCl}_{3}\right) \delta 11.90$ (d, 1H, NH), $10.10(\mathrm{~s}, 1 \mathrm{H}, \mathrm{NH}), 8.07$ (d, 1H, NCH), 7.33-7.18 (m, 6H, $\left.H_{\text {Aryl }}\right), 4.25\left(\mathrm{q}, 2 \mathrm{H}, \mathrm{CH}_{2}\right)$, $3.20\left(\mathrm{~m}_{7}, 2 \mathrm{H}, \mathrm{CH}\right), 3.19\left(\mathrm{~m}_{7}, 2 \mathrm{H}, \mathrm{CH}\right), 1.31\left(\mathrm{t}, 3 \mathrm{H}, \mathrm{CH}_{3}\right), 1.23\left(\mathrm{~d}, 24 \mathrm{H}, \mathrm{CH}_{3}\right) .{ }^{13} \mathrm{C} \mathrm{NMR}\left(\mathrm{CDCl}_{3}\right)$ $\delta 169.3,169.1,159.7,146.7,144.4,136.5,132.3,128.2,128.1,124.4,123.7,91.0,60.4,29.4$, 28.9, 24.1, 14.8. $\mathrm{EI}^{+}-\mathrm{HR}-\mathrm{MS}: \mathrm{m} / z 478.3195$ (calcd.), found 478.3201 for $\mathrm{C}_{30} \mathrm{H}_{42} \mathrm{~N}_{2} \mathrm{O}_{3}$, $\delta=0.6 \mathrm{mDa}$. M.p. $182 \cdot 0-182.7^{\circ} \mathrm{C}$.

Ethyl-3-[(2,6-diethylphenyl)amino]-2-\{[(4-methylphenyl)amino]carbonyl\}acrylate (IXc), A mixture of diethyl $\{[(2,6$-diethylphenyl)amino]methylene $\}$ malonate (Vib) $(1.20 \mathrm{~g}, 4 \mathrm{mmol})$ and p-toluidine $(0.44 \mathrm{~g}, 4 \mathrm{mmol})$ was heated at $135{ }^{\circ} \mathrm{C}$ for $30 \mathrm{~h}$ while the produced ethanol was 
distilled out of the reaction mixture. The mixture was cooled and $10 \mathrm{ml}$ of hexane were added while vigorously stirring. The mixture was stirred $30 \mathrm{~min}$ in an ice-bath and the formed precipitate was filtered and purified by column chromatography (on silica, ethylacetate:hexane $=$ 1:4). Yield: $0.67 \mathrm{~g}(2 \mathrm{mmol}, 46 \%) .{ }^{1} \mathrm{H} \mathrm{NMR}\left(\mathrm{CDCl}_{3}\right) \delta 11.78(\mathrm{~d}, 1 \mathrm{H}, \mathrm{NH}), 10.89(\mathrm{~s}, 1 \mathrm{H}$, $\mathrm{C}(\mathrm{O}) \mathrm{N} H), 8.08(\mathrm{~d}, 1 \mathrm{H}, \mathrm{C}=\mathrm{CH}), 7.53\left(\mathrm{~d}, 2 \mathrm{H}, H_{\text {Aryl }}\right), 7.24-7.13\left(\mathrm{~m}, 5 \mathrm{H}, H_{\text {Aryl }}\right), 4.23\left(\mathrm{q}, 2 \mathrm{H}, \mathrm{OC} H_{2}\right)$, $2.69\left(\mathrm{q}, 4 \mathrm{H}, \mathrm{CH}_{2}\right), 2.33\left(\mathrm{~s}, 3 \mathrm{H}, \mathrm{CH}_{3}\right), 1.30(\mathrm{t}, 3 \mathrm{H}, \mathrm{CH}), 1.25\left(\mathrm{t}, 6 \mathrm{H}, \mathrm{CH}_{3}\right) .{ }^{13} \mathrm{C} \mathrm{NMR}\left(\mathrm{CDCl}_{3}\right)$ $\delta 168.7,167.6,158.8,139.5,137.2,135.9,133.1,129.4,127.6,127.1,120.6,91.0,60.1,24.9$, 20.9, 14.9, 14.5. $\mathrm{EI}^{+}-\mathrm{HR}-\mathrm{MS}: \mathrm{m} / z 380.2100$ (calcd.), found 380.2075 for $\mathrm{C}_{23} \mathrm{H}_{28} \mathrm{~N}_{2} \mathrm{O}_{3}$, $\delta=2.5 \mathrm{mDa}$

Ethyl-2-\{[(2,6-dimethylphenyl)amino]carbonyl\}-3-[(4-methylphenyl)amino]acrylate (IXd), Method A: A mixture of equimolar amounts of diethyl \{[(4-methylphenyl)amino $]-$ methylene $\}$ malonate (VIa) $(2.00 \mathrm{~g}, 7.2 \mathrm{mmol})$ and 2,6-dimethylaniline $(0.88 \mathrm{~g}, 7.2 \mathrm{mmol})$ was heated at $155{ }^{\circ} \mathrm{C}$ for $16 \mathrm{~h}$ while the produced ethanol was distilled out of the reaction mixture. Then the mixture was cooled and $40 \mathrm{ml}$ of hexane were added; the mixture was further stirred $1 \mathrm{~h}$ in an ice-bath and finally stored at $-30{ }^{\circ} \mathrm{C}$. The formed colorless crystals were filtered. Yield: 2.44 g (6.9 mmol, $96 \%)$. Method B: A mixture of ethyl-3-[(2,6-dimethylphenyl)amino]3-oxopropanoate (VId) $(1.25 \mathrm{~g}, 5.3 \mathrm{mmol}), \quad$-toluidine $(0.60 \mathrm{~g}, 5.5 \mathrm{mmol})$ and triethyl orthoformate $(0.83 \mathrm{~g}, 5.5 \mathrm{mmol})$ was heated at $160{ }^{\circ} \mathrm{C}$ for $4 \mathrm{~h}$ while the produced ethanol was distilled out of the reaction mixture. The mixture was then cooled and mixed with $10 \mathrm{ml}$ of hexane in an ice-bath for $30 \mathrm{~min}$. A white solid precipitated, which was filtered and recrystallized from ethylacetate/hexane. Yield: $0.94 \mathrm{~g}(2.7 \mathrm{mmol}, 50 \%) .{ }^{1} \mathrm{H} \mathrm{NMR}\left(\mathrm{CDCl}_{3}\right)$ $\delta 12.38(\mathrm{~d}, 1 \mathrm{H}, \mathrm{NH}), 10.20(\mathrm{~s}, 1 \mathrm{H}, \mathrm{NH}), 8.56(\mathrm{~d}, 1 \mathrm{H}, \mathrm{C}=\mathrm{CH}), 7.16\left(\mathrm{~d}, 2 \mathrm{H}, H_{\text {Aryl }}\right), 7.10(\mathrm{sb}, 3 \mathrm{H}$, $\left.H_{\text {Aryl }}\right), 7.02\left(\mathrm{~d}, 2 \mathrm{H}, H_{\text {Aryl }}\right), 4.31\left(\mathrm{q}, 2 \mathrm{H}, \mathrm{OCH}_{2}\right), 2.33\left(\mathrm{~s}, 3 \mathrm{H}, \mathrm{CH}_{3}\right), 2.29\left(\mathrm{~s}, 6 \mathrm{H}, \mathrm{CH}_{3}\right), 1.39(\mathrm{t}, 3 \mathrm{H}$, $\left.\mathrm{CH}_{3}\right) .{ }^{13} \mathrm{C} \mathrm{NMR}\left(\mathrm{CDCl}_{3}\right) \delta 168.6,167.6,151.1,137.1,135.6,134.5,134.4,130.3,128.0,126.8$, 117.1, 99.6, 60.2, 20.9, 18.7, 14.6. EI+HR-MS: m/z 352.1787 (calcd.), found 352.1765 for $\mathrm{C}_{21} \mathrm{H}_{24} \mathrm{~N}_{2} \mathrm{O}_{3}, \delta=2.2 \mathrm{mDa}$. M.p. $128.1-129.3^{\circ} \mathrm{C}$. 
(IXe): A mixture of ethyl-3-[(2,6-diisopropylphenyl)amino]-3-oxopropanoate (VIe) (2.10 g, $7 \mathrm{mmol})$, 2,6-dimethylaniline $(0.90 \mathrm{~g}, 7 \mathrm{mmol})$ and triethyl orthoformate $(1.10 \mathrm{~g}, 7 \mathrm{mmol})$ was heated at $130{ }^{\circ} \mathrm{C}$ for $5 \mathrm{~h}$ while the produced ethanol was distilled out of the reaction mixture. An oil resulted, which solidified at $\mathrm{r}$. t.. The white solid was crushed in a mortar, mixed with $50 \mathrm{ml}$ of cold hexane, then stirred for $30 \mathrm{~min}$ and was finally filtered. Yield: $2.07 \mathrm{~g}(5 \mathrm{mmol}, 70 \%) .{ }^{1} \mathrm{H}$ $\operatorname{NMR}\left(\mathrm{CDCl}_{3}\right) \delta 11.90(\mathrm{~d}, 1 \mathrm{H}, \mathrm{NH}), 10.12(\mathrm{~s}, 1 \mathrm{H}, \mathrm{NH}), 8.19(\mathrm{~d}, 1 \mathrm{H}, \mathrm{C}=\mathrm{CH}), 7.33-7.09(\mathrm{~m}, 6 \mathrm{H}$, $\left.H_{\text {Aryl }}\right), 4.27\left(\mathrm{q}, 2 \mathrm{H}, \mathrm{OCH}_{2}\right), 3.20\left(\mathrm{~m}_{7}, 2 \mathrm{H}, \mathrm{CH}\right), 2.33\left(\mathrm{~s}, 6 \mathrm{H}, \mathrm{CH}_{3}\right), 1.34\left(\mathrm{t}, 3 \mathrm{H}, \mathrm{CH}_{3}\right), 1.25(\mathrm{bd}$, $\left.12 \mathrm{H}, \mathrm{CH}_{3}\right) .{ }^{13} \mathrm{C}$ NMR $\left(\mathrm{CDCl}_{3}\right) \delta 168.8,168.7,158.0,146.2,138.2,132.3,131.8,129.0,127.8$, 126.5, 123.3, 90.7, 60.0, 28.9, 24.2, 23.2, 18.6, 14.5. EI'-HR-MS: $m / z$ 422.2569 (calcd.), found 422.2599 for $\mathrm{C}_{26} \mathrm{H}_{34} \mathrm{~N}_{2} \mathrm{O}_{3}, \delta=3.0$ mDa. M.p. $120.3-121.0^{\circ} \mathrm{C}$.

N,N'-Bis(1-pyridin-2-ylethyl)malonamide (IXf): Diethyl malonate (1.05 g, $6.5 \mathrm{mmol})$ was stirred under argon with an excess of 1-pyridin-2-ylethanamine $(2.05 \mathrm{~g}, 16.8 \mathrm{mmol})$ at $170{ }^{\circ} \mathrm{C}$ for $10 \mathrm{~h}$. The mixture was allowed to cool to ambient temperature and the ethanol was removed under reduced pressure. The product was used for the following step without any further purification. Yield: $1.93 \mathrm{~g}(6.2 \mathrm{mmol}, 95 \%) .{ }^{1} \mathrm{H} \mathrm{NMR}\left(\mathrm{CDCl}_{3}\right) \delta 8.50(\mathrm{~d}, 2 \mathrm{H}, H \mathrm{Pyr}), 8.13(\mathrm{~d}, 2 \mathrm{H}$, NH), 7.60 (dt, 2H, HPyr), 7.22 (d, 2H, HPyr), 7.14 (dt, 2H, HPyr), 5.11 (p, 2H, CH), 3.28 (s, 2H, $\left.\mathrm{CH}_{2}\right), 1.46\left(\mathrm{~d}, 6 \mathrm{H}, \mathrm{CH}_{3}\right) .{ }^{13} \mathrm{C} \mathrm{NMR}\left(\mathrm{CDCl}_{3}\right) \delta 166.6,161.0,149.1,136.8,122.3,121.1,50.3$, 43.3, 22.1. $\mathrm{EI}^{+}-\mathrm{MS} m / z=312\left(\mathrm{M}^{+}\right)$.

Ethyl-2-\{ethoxy[(2,6-dimethylphenyl)imino]methyl\}-3-[(2,6-dimethylphenyl)amino]acrylate (Xa): A mixture of 3-[(2,6-dimethylphenyl)amino]-2-\{[(2,6-dimethylphenyl)amino]carbonyl $\}$ acrylate $(\mathbf{I X a})(3.00 \mathrm{~g}, 8.2 \mathrm{mmol})$ and a $1.0 \mathrm{M}$ solution of triethyloxonium tetrafluoroborate in dichloromethane $(12.34 \mathrm{~g}, 9.3 \mathrm{mmol})$ was stirred $7 \mathrm{~d}$ at ambient temperature. The solvent was removed in vacuum and the residue was washed twice with abs. diethylether. Then the residue was taken up in $40 \mathrm{ml}$ of abs. diethylether and cooled to $0{ }^{\circ} \mathrm{C}$. Triethylamine $(1.3 \mathrm{ml}$, $9.3 \mathrm{mmol}$ ) was slowly added and the mixture was stirred at $\mathrm{r}$. t. for $2 \mathrm{~h}$. The organic phase was separated and the residue was washed three times with abs. diethylether. The diethylether 
solutions were collected and dried over $\mathrm{Na}_{2} \mathrm{SO}_{4}$. The solvent was removed under reduced pressure. A yellow oil was obtained, which was mixed with $10 \mathrm{ml}$ of hexane and stored overnight at $-30{ }^{\circ} \mathrm{C}$ : the formed precipitate was filtered and recrystallized in big colorless rhomboidal crystals from hexane:ethylacetate $=4: 1$. Yield: $2.11 \mathrm{~g}(5.3 \mathrm{mmol}, 65 \%) .{ }^{1} \mathrm{H}$ NMR $\left(\mathrm{CDCl}_{3}\right) \delta 9.74(\mathrm{~d}, 1 \mathrm{H}, \mathrm{N} H), 7.01\left(\mathrm{sb}, 3 \mathrm{H}, H_{\text {Aryl }}\right), 6.94\left(\mathrm{~d}, 2 \mathrm{H}, H_{\text {Aryl }}\right), 6.82(\mathrm{~d}, 1 \mathrm{H}, \mathrm{C}=\mathrm{C} H), 6.78$ $\left(\mathrm{t}, 1 \mathrm{H}, H_{\text {Aryl }}\right), 4.36\left(\mathrm{q}, 2 \mathrm{H}, \mathrm{OCH}_{2}\right), 4.08\left(\mathrm{q}, 2 \mathrm{H}, \mathrm{OCH}_{2}\right), 2.09\left(\mathrm{~s}, 6 \mathrm{H}, \mathrm{CH}_{3}\right), 2.01\left(\mathrm{~s}, 6 \mathrm{H}, \mathrm{CH}_{3}\right)$, $1.39\left(\mathrm{t}, 3 \mathrm{H}, \mathrm{CH}_{3}\right), 1.25\left(\mathrm{t}, 3 \mathrm{H}, \mathrm{CH}_{3}\right) .{ }^{13} \mathrm{C} \mathrm{NMR}\left(\mathrm{CDCl}_{3}\right) \delta 157.0,153.5,146.2,138.4,133.1$, 129.0, 128.4, 128.3, 126.5, 122.4, 100.0, 93.2, 62.2, 59.9, 19.0, 18.4, 14.8, 14.6. EI ${ }^{+}-\mathrm{HR}-\mathrm{MS}:$ $m / z 394.2256$ (calcd.), found 394.2257 for $\mathrm{C}_{24} \mathrm{H}_{30} \mathrm{~N}_{2} \mathrm{O}_{3}, \delta=0.1 \mathrm{mDa}$. M.p. 69.6-71.0 ${ }^{\circ} \mathrm{C}$.

Ethyl-2-\{ethoxy[(2,6-diisopropylphenyl)imino]methyl\}-3-[(2,6-diisopropylphenyl)amino]acrylate (Xb): A mixture of ethyl-3-[(2,6-diisopropylphenyl)amino]-2-\{[(2,6-diisopropylphenyl)amino]carbonyl $\}$ acrylate (IXb) $(4.00 \mathrm{~g}, 8 \mathrm{mmol})$ and a $1.0 \mathrm{M}$ solution of triethyloxonium tetrafluoroborate in dichloromethane $(12.45 \mathrm{~g}, 9 \mathrm{mmol})$ was stirred $9 \mathrm{~d}$ at ambient temperature. The solvent was removed in vacuum and the residue was washed twice with abs. diethylether. Then the residue was taken up in $30 \mathrm{ml}$ of abs. diethylether and cooled to $0{ }^{\circ} \mathrm{C}$. Triethylamine $(1.3 \mathrm{ml}, 9 \mathrm{mmol})$ was slowly added and the mixture was stirred at $\mathrm{r}$. t. for $2 \mathrm{~h}$. The organic phase was separated and the residue was washed three times with abs. diethylether. The diethylether solutions were collected, dried over $\mathrm{Na}_{2} \mathrm{SO}_{4}$, and the solvent was removed under reduced pressure. An oil was obtained which crystallized slowly. This solid was filtered. Yield: $2.57 \mathrm{~g}$ $(5 \mathrm{mmol}, 60 \%) .{ }^{1} \mathrm{H}$ NMR $\left(\mathrm{CDCl}_{3}\right) \delta 9.63(\mathrm{~d}, 1 \mathrm{H}, \mathrm{NH}), 7.26-6.89\left(\mathrm{~m}, 6 \mathrm{H}, H_{\text {Aryl }}\right), 6.61(\mathrm{~d}, 1 \mathrm{H}$, $\mathrm{C}=\mathrm{CH}), 4.34\left(\mathrm{q}, 2 \mathrm{H}, \mathrm{OCH}_{2}\right), 4.18\left(\mathrm{q}, 2 \mathrm{H}, \mathrm{OCH}_{2}\right), 2.97\left(\mathrm{~m}_{7}, 2 \mathrm{H}, \mathrm{CH}\right), 2.74\left(\mathrm{~m}_{7}, 2 \mathrm{H}, \mathrm{CH}\right), 1.38(\mathrm{t}$, $\left.3 \mathrm{H}, \mathrm{CH}_{3}\right), 1.30\left(\mathrm{t}, 3 \mathrm{H}, \mathrm{CH}_{3}\right), 1.12\left(\mathrm{~d}, 6 \mathrm{H}, \mathrm{CH}_{3}\right), 1.05\left(\mathrm{~d}, 12 \mathrm{H}, \mathrm{CH}_{3}\right), 1.02\left(\mathrm{~d}, 6 \mathrm{H}, \mathrm{CH}_{3}\right) .{ }^{13} \mathrm{C} \mathrm{NMR}$ $\left(\mathrm{CDCl}_{3}\right) \delta 168.8,157.4,155.9,154.9,144.9,143.3,137.5,127.9,123.5,122.8,122.7,91.9,61.8$ 59.6, 28.0, 24.1, 23.4, 22.3, 14.5, 14.4. EI $^{+}$-HR-MS: $m / z$ 506.3508 (calcd.), found 506.3554 for $\mathrm{C}_{32} \mathrm{H}_{46} \mathrm{~N}_{2} \mathrm{O}_{3}, \delta=4.6 \mathrm{mDa}$. M.p. $102.8-103.3^{\circ} \mathrm{C}$.

Ethyl-3-[(2,6-diethylphenyl)amino]-2-\{ethoxy[(4-methylphenyl)imino]methyl\}acrylate (Xc): A mixture of ethyl-3-[(2,6-diethylphenyl)amino]-2-\{[(4-methylphenyl)amino]carbonyl $\}$ acrylate 
(IXc) $(0.60 \mathrm{~g}, 2 \mathrm{mmol})$ and a $1.0 \mathrm{M}$ solution of triethyloxonium tetrafluoroborate in dichloromethane $(2.35 \mathrm{~g}, 2 \mathrm{mmol})$ was stirred $35 \mathrm{~d}$ at ambient temperature. The solvent was evaporated in vacuum. The residue was taken up in $20 \mathrm{ml}$ of abs. diethylether, cooled to $0{ }^{\circ} \mathrm{C}$, and washed with abs. diethylether. Triethylamine $(0.5 \mathrm{ml})$ was slowly added and the mixture was stirred at r. t. for $2 \mathrm{~h}$. The ether phase was separated and the residue was washed with abs. ether. The ether solutions were collected and the solvent was evaporated in vacuum. The resulting solid was recrystallized from diethylether. Yield $0.37 \mathrm{~g}(0.9 \mathrm{mmol}, 57 \%) .{ }^{1} \mathrm{H} \mathrm{NMR}\left(\mathrm{CDCl}_{3}\right) \delta 9.58(\mathrm{~d}$, $1 \mathrm{H}, \mathrm{NH}), 7.18-6.89\left(\mathrm{~m}, 5 \mathrm{H}, H_{\text {Aryl }}\right), 6.78(\mathrm{~d}, 1 \mathrm{H}, \mathrm{C}=\mathrm{CH}), 6.71\left(\mathrm{~d}, 2 \mathrm{H}, H_{\text {Aryl }}\right), 4.29\left(\mathrm{q}, 2 \mathrm{H}, \mathrm{OC} H_{2}\right)$, $4.01\left(\mathrm{q}, 2 \mathrm{H}, \mathrm{OCH}_{2}\right), 2.44\left(\mathrm{q}, 4 \mathrm{H}, \mathrm{CH}_{2}\right), 2.28\left(\mathrm{~s}, 3 \mathrm{H}, \mathrm{CH}_{3}\right), 1.35\left(\mathrm{t}, 3 \mathrm{H}, \mathrm{CH}_{3}\right), 1.20\left(\mathrm{t}, 3 \mathrm{H}, \mathrm{CH}_{3}\right)$, $1.13\left(\mathrm{t}, 6 \mathrm{H}, \mathrm{CH}_{3}\right) .{ }^{13} \mathrm{C} \mathrm{NMR}\left(\mathrm{CDCl}_{3}\right) \delta 168.5,159.5,154.6,147.1,139.9,137.5,131.8,129.6$, 127.4, 127.2, 122.0, 93.0, 62.3, 60.0, 25.0, 21.2, 15.0, 14.7, 14.6. - EI+HR-MS: m/z 408.2413 (calcd.), found 408.2433 for $\mathrm{C}_{25} \mathrm{H}_{32} \mathrm{~N}_{2} \mathrm{O}_{3}, \delta=2.0 \mathrm{mDa}$.

Ethyl-2-\{ethoxy[(2,6-dimethylphenyl)imino]methyl\}-3-[(4-methylphenyl)amino]acrylate (Xd): A mixture of ethyl-2-\{[(2,6-dimethylphenyl)amino]carbonyl $\}-3-[(4-m e t h y l p h e n y l) a m i n o]-$ acrylate (IXd) $(1.60 \mathrm{~g}, 4.5 \mathrm{mmol})$ and a $1.0 \mathrm{M}$ solution of triethyloxonium tetrafluoroborate in dichloromethane $(6.68 \mathrm{~g}, 5.0 \mathrm{mmol})$ was stirred $12 \mathrm{~d}$ at ambient temperature. The solvent was removed in vacuum and the residue was washed twice with abs. diethylether. Then, the residue was taken up in $40 \mathrm{ml}$ of abs. diethylether and cooled to $0{ }^{\circ} \mathrm{C}$. Triethylamine $(0.7 \mathrm{ml}, 5.0 \mathrm{mmol})$ was slowly added and the mixture was stirred at r. t. for $2 \mathrm{~h}$. The organic phase was separated and the residue was washed three times with abs. diethylether. The diethylether solutions were collected, dried over $\mathrm{Na}_{2} \mathrm{SO}_{4}$, and the solvent was removed under reduced pressure. A yellow oil remained, which was mixed with $10 \mathrm{ml}$ of hexane and stored overnight at $-30{ }^{\circ} \mathrm{C}$ : small yellow crystals formed and were filtered. Yield: $0.30 \mathrm{~g}(0.8 \mathrm{mmol}, 18 \%) .{ }^{1} \mathrm{H}$ NMR $\left(\mathrm{CDCl}_{3}\right) \delta 10.06(\mathrm{~d}$, $1 \mathrm{H}, \mathrm{NH}), 7.14(\mathrm{~d}, 1 \mathrm{H}, \mathrm{C}=\mathrm{CH}), 7.05\left(\mathrm{~d}, 2 \mathrm{H}, H_{\text {Aryl }}\right), 7.00\left(\mathrm{~d}, 2 \mathrm{H}, H_{\text {Aryl }}\right), 6.82\left(\mathrm{t}, 1 \mathrm{H}, H_{\text {Aryl }}\right), 6.54(\mathrm{~d}$, $\left.2 \mathrm{H}, H_{\text {Aryl }}\right), 4.39$ (q, 2H, OCH $\left.H_{2}\right), 4.12\left(\mathrm{q}, 2 \mathrm{H}, \mathrm{OCH}_{2}\right), 2.28\left(\mathrm{~s}, 3 \mathrm{H}, \mathrm{CH}_{3}\right), 2.13\left(\mathrm{~s}, 6 \mathrm{H}, \mathrm{CH}_{3}\right), 1.42$ $\left(\mathrm{t}, 3 \mathrm{H}, \mathrm{CH}_{3}\right), 1.26\left(\mathrm{t}, 3 \mathrm{H}, \mathrm{CH}_{3}\right) .{ }^{13} \mathrm{C} \mathrm{NMR}\left(\mathrm{CDCl}_{3}\right) \delta 168.2,157.0,146.8,146.1,137.6,133.4$, 
$130.2,128.1,127.9,122.2,116.5,94.1,61.9,59.8,20.7,18.5,14.5,14.3 . \mathrm{EI}^{+}-\mathrm{HR}-\mathrm{MS}:$ $m / z 380.2100$ (calcd.), found 380.2067 for $\mathrm{C}_{23} \mathrm{H}_{28} \mathrm{~N}_{2} \mathrm{O}_{3}, \delta=3.3 \mathrm{mDa}$. M.p. 60.6-61.5 ${ }^{\circ} \mathrm{C}$.

Ethyl-2-[[(2,6-diisopropylphenyl)imino](ethoxy)methyl]-3-[(2,6-dimethylphenyl)amino]-

acrylate (Xe): A mixture of ethyl-2-\{[(2,6-diisopropylphenyl)amino]carbonyl $\}-3-[(2,6-$ dimethylphenyl)amino]acrylate (IXe) $(1.43 \mathrm{~g}, 3 \mathrm{mmol})$ and a $1.0 \mathrm{M}$ solution of triethyloxonium tetrafluoroborate in dichloromethane $(4.97 \mathrm{~g}, 4 \mathrm{mmol})$ was stirred $30 \mathrm{~d}$ at ambient temperature. The solvent was removed in vacuum and the residue was washed twice with abs. diethylether. The residue was taken up in $20 \mathrm{ml}$ of abs. diethylether and cooled to $0{ }^{\circ} \mathrm{C}$. Triethylamine $(0.6 \mathrm{ml})$ was slowly added and the mixture was stirred at $\mathrm{r}$. t. for $2 \mathrm{~h}$. The organic phase was separated and the residue was washed three times with abs. diethylether. The diethylether solutions were collected and the solvent was removed under reduced pressure. Small colorless crystals slowly precipitated from the oil. The crystals were filtered and washed with cold diethylether. Yield: $0.40 \mathrm{~g}(0.9 \mathrm{mmol}, 26 \%) .{ }^{1} \mathrm{H}$ NMR $\left(\mathrm{CDCl}_{3}\right) \delta 9.62(\mathrm{~d}, 1 \mathrm{H}, \mathrm{NH}), 7.10-6.90$ $\left(\mathrm{m}, 6 \mathrm{H}, H_{\text {Aryl }}\right), 6.60(\mathrm{~d}, 1 \mathrm{H}, \mathrm{C}=\mathrm{CH}), 4.34\left(\mathrm{q}, 2 \mathrm{H}, \mathrm{OCH}_{2}\right), 4.20\left(\mathrm{q}, 2 \mathrm{H}, \mathrm{OCH} H_{2}\right), 2.98\left(\mathrm{~m}_{7}, 2 \mathrm{H}, \mathrm{CH}\right)$, $1.88\left(\mathrm{~s}, 6 \mathrm{H}, \mathrm{CH}_{3}\right), 1.38\left(\mathrm{t}, 3 \mathrm{H}, \mathrm{CH}_{3}\right), 1.31\left(\mathrm{t}, 3 \mathrm{H}, \mathrm{CH}_{3}\right), 1.12\left(\mathrm{~d}, 6 \mathrm{H}, \mathrm{CH}_{3}\right), 1.04\left(\mathrm{~d}, 6 \mathrm{H}, \mathrm{CH}_{3}\right) .{ }^{13} \mathrm{C}$ NMR $\left(\mathrm{CDCl}_{3}\right) \delta 168.8,156.0,153.6,143.4,138.0,137.8,133.0,128.4,126.3,123.0,122.6$, 92.2, 61.7, 59.7, 27.9, 24.1, 22.3, 17.8, 14.5, 14.4. $\mathrm{EI}^{+}-\mathrm{HR}-\mathrm{MS}: \mathrm{m} / z 450.2882$ (calcd.), found 450.2905 for $\mathrm{C}_{28} \mathrm{H}_{38} \mathrm{~N}_{2} \mathrm{O}_{3}, \delta=2.3 \mathrm{mDa}$.

1-Methyl-3-[(1-methylimidazo[1,5-a]pyridin-3-yl)methyl]imidazo[1,5-a]pyridine (Xf): A mixture containing the bisamide IXf $(6.2 \mathrm{mmol}, 1.93 \mathrm{~g})$, an excess of phosphorus oxychloride $(10 \mathrm{ml}, 100 \mathrm{mmol})$ and $15 \mathrm{ml}$ of toluene were stirred for $4 \mathrm{~h}$ under argon while refluxing. The excess of $\mathrm{POCl}_{3}$ and the solvent were removed under reduced pressure. $50 \mathrm{ml}$ of distilled water were added, the mixture was neutralised with sodium hydroxide and extracted with ethyl acetate. The extracts were collected and the solvent was removed. The resulting solid was recrystallized from hot ethyl acetate. Brown crystals precipitated, which were filtered and dried. Yield: $1.14 \mathrm{~g}$ (4.1 mmol, 67\%). ${ }^{1} \mathrm{H}$ NMR $\left(\mathrm{CDCl}_{3}\right) \delta 8.13(\mathrm{~d}, 2 \mathrm{H}, H \mathrm{Pyr}), 7.20$ (d, 2H, HPyr), 6.50 (dt, 2H, 
HPyr), 6.41 (dt, 2H, HPyr), 4.81 (s, 2H, $\mathrm{CH}_{2}$ ), 2.43 (s, 6H, $\mathrm{CH}_{3}$ ). EI ${ }^{+}-\mathrm{HR}-\mathrm{MS}: m / z$ 276.1375 (calcd.), found 276.1341 for $\mathrm{C}_{17} \mathrm{H}_{16} \mathrm{~N}_{4}, \mathrm{~d}=3.4 \mathrm{mDa}$.

N,N'-bis(2,6-diisopropylphenyl)malonamide (XI): A mixture of diethyl malonate $(10.10 \mathrm{~g}$, $0.062 \mathrm{~mol})$ and 2,6-diisopropylaniline $(25.00 \mathrm{~g}, 0.127 \mathrm{~mol})$ was heated at $160{ }^{\circ} \mathrm{C}$ for $10 \mathrm{~h}$, while the produced ethanol was distilled out of the reaction mixture. The mixture was cooled to $\mathrm{r}$. $\mathrm{t}$. and the resulting powder was mixed with $200 \mathrm{ml}$ of cold hexane and stirred for $1 \mathrm{~h}$. The microcrystalline pale-pink powder was filtered and washed with hexane. Yield: $23.79 \mathrm{~g}$ (56 mmol, 90\%). ${ }^{1} \mathrm{H}$ NMR $\left(\mathrm{CDCl}_{3}\right) \delta 8.48(\mathrm{~s}, 2 \mathrm{H}, \mathrm{N} H), 7.20\left(\mathrm{t}, 1 \mathrm{H}, H_{\text {Aryl }}\right), 7.10\left(\mathrm{~d}, 4 \mathrm{H}, H_{\text {Aryl }}\right)$, $3.62\left(\mathrm{~s}, 2 \mathrm{H}, \mathrm{CH}_{2}\right), 2.98\left(\mathrm{~m}_{7}, 4 \mathrm{H}, \mathrm{CH}\right), 1.11\left(\mathrm{~d}, 24 \mathrm{H}, \mathrm{CH}_{3}\right) .{ }^{13} \mathrm{C} \mathrm{NMR}\left(\mathrm{CDCl}_{3}\right) \delta 167.1,145.9$, 130.7, 128.5, 123.5, 42.7, 28.9, 23.6. $\mathrm{EI}^{+}$-HR-MS: $m / z 422.2933$ (calcd), found 422.2910 for $\mathrm{C}_{27} \mathrm{H}_{38} \mathrm{~N}_{2} \mathrm{O}_{2}, \delta=2.3 \mathrm{mDa}$. M.p. $270.3-271.7^{\circ} \mathrm{C}$.

Ethyl-N-(2,6-diisopropylphenyl)-3-[(2,6-diisopropylphenyl)amino]-3-oxopropanimidoate(XII): A mixture of $N, N^{\prime}$-bis(2,6-diisopropylphenyl)malonamide (XI) $(10.00 \mathrm{~g}, 24 \mathrm{mmol})$ and a $1.0 \mathrm{M}$ solution of triethyloxonium tetrafluoroborate in dichloromethane $(74.08 \mathrm{~g}, 56 \mathrm{mmol})$ was stirred $20 \mathrm{~d}$ at ambient temperature. The solvent was evaporated in vacuum and the residue was washed with $160 \mathrm{ml}$ of abs. diethylether and filtered. Then it was taken up in $60 \mathrm{ml}$ of abs. diethylether, cooled to $0{ }^{\circ} \mathrm{C}$ and triethylamine $(7.5 \mathrm{ml}, 54 \mathrm{mmol})$ was slowly added. The mixture was stirred at r. t. for $2 \mathrm{~h}$ and then it was filtered. The ether phases were collected and the solvent was removed under reduced pressure; a white solid precipitated, which was recrystallized from toluene:hexane = 3:1. Yield: $6.97 \mathrm{~g}(15 \mathrm{mmol}, 64 \%) .{ }^{1} \mathrm{H} \mathrm{NMR}\left(\mathrm{CDCl}_{3}\right) \delta 7.59(\mathrm{~s}, 1 \mathrm{H}, \mathrm{NH}), 7.34-7.02(\mathrm{~m}, 6 \mathrm{H}$, $\left.H_{\text {Aryl }}\right), 4.50\left(\mathrm{q}, 2 \mathrm{H}, \mathrm{OCH}_{2}\right), 3.25\left(\mathrm{~s}, 2 \mathrm{H}, \mathrm{CH}_{2}\right), 3.08\left(\mathrm{~m}_{7}, 2 \mathrm{H}, \mathrm{CH}\right), 2.87\left(\mathrm{~m}_{7}, 2 \mathrm{H}, \mathrm{CH}\right), 1.47(\mathrm{t}, 3 \mathrm{H}$, $\left.\mathrm{CH}_{3}\right), 1.21\left(\mathrm{~d}, 12 \mathrm{H}, \mathrm{CH}_{3}\right), 1.20\left(\mathrm{~d}, 6 \mathrm{H}, \mathrm{CH}_{3}\right), 1.16\left(\mathrm{~d}, 6 \mathrm{H}, \mathrm{CH}_{3}\right) .{ }^{13} \mathrm{C} \mathrm{NMR}\left(\mathrm{CDCl}_{3}\right) \delta 165.4$, $154.9,146.2,137.8,131.0,128.6,123.8,123.7,123.6,123.2,62.6,38.4,28.8,28.3,23.4,22.7$, 14.5. $\mathrm{EI}^{+}$-HR-MS: $m / z 450.3246$ (calcd.), found 450.3172 for $\mathrm{C}_{29} \mathrm{H}_{42} \mathrm{~N}_{2} \mathrm{O}_{2}, \quad \delta=7.4 \mathrm{mDa}$. M.p. $158.2-159.9^{\circ} \mathrm{C}$.

[1] La Forge, F. B. J. Am. Chem. Soc. 1928, 50, 2477-2483. 
Oligomer distribution in oligomerization experiments

Table S1. Yield (\%) of oligomers in the experiments 32, 33, 35-42.

\begin{tabular}{l|rrrrrrrrrr}
\hline Fraction (\%) & \multicolumn{1}{|c}{32} & \multicolumn{1}{c}{33} & \multicolumn{1}{c}{35} & \multicolumn{1}{c}{36} & \multicolumn{1}{c}{37} & \multicolumn{1}{c}{38} & \multicolumn{1}{c}{39} & \multicolumn{1}{c}{40} & \multicolumn{1}{c}{41} & \multicolumn{1}{c}{42} \\
\hline $\mathrm{C}_{6}$ & 93.0 & 84.8 & 87.5 & 87.6 & 95.2 & 92.5 & 89.6 & 91.6 & 95.7 & 91.5 \\
$\mathrm{C}_{9}$ & 6.7 & 5.5 & 6.1 & 5.5 & 3.2 & 4.4 & 4.1 & 4.1 & 2.8 & 5.5 \\
$\mathrm{C}_{12}$ & 0.2 & 5.1 & 3.9 & 4.5 & 1.7 & 1.9 & 3.5 & 2.7 & 1.5 & 2.2 \\
$\mathrm{C}_{15}$ & - & 2.0 & 0.8 & 1.1 & - & 0.5 & 1.2 & 0.9 & - & 0.3 \\
$\mathrm{C}_{18}$ & - & 1.8 & 1.6 & 1.1 & - & 0.7 & 1.1 & 0.7 & - & 0.5 \\
$\mathrm{C}_{21}$ & - & 0.5 & - & 0.1 & - & 0.3 & - & - & - & - \\
$\mathrm{C}_{24}$ & - & 0.3 & - & 0.1 & - & - & - & - & - & - \\
\hline
\end{tabular}

Table S2. Yield (\%) of oligomers in the experiments 43, 44, 46-50.

\begin{tabular}{l|rrrrrrr}
\hline Fraction (\%) & \multicolumn{1}{c}{43} & \multicolumn{1}{c}{44} & \multicolumn{1}{c}{46} & \multicolumn{1}{c}{47} & \multicolumn{1}{c}{48} & \multicolumn{1}{c}{49} & \multicolumn{1}{c}{50} \\
\hline $\mathrm{C}_{6}$ & 89.8 & 86.0 & 92.2 & 92.0 & 95.2 & 91.4 & 84.7 \\
$\mathrm{C}_{9}$ & 5.5 & 6.1 & 4.5 & 4.2 & 3.3 & 3.6 & 7.5 \\
$\mathrm{C}_{12}$ & 3.0 & 4.6 & 2.2 & 2.4 & 1.2 & 2.7 & 4.7 \\
$\mathrm{C}_{15}$ & 0.9 & 1.3 & 0.5 & 0.7 & 0.1 & 0.9 & 1.4 \\
$\mathrm{C}_{18}$ & 0.8 & 1.3 & 0.6 & 0.7 & 0.2 & 0.9 & 1.7 \\
$\mathrm{C}_{21}$ & - & 0.3 & - & - & - & 0.3 & - \\
$\mathrm{C}_{24}$ & - & 0.3 & - & - & - & 0.2 & - \\
\hline
\end{tabular}

